\title{
BMJ open Sexual and geographic organisation of men who have sex with men in a large East African city: opportunities for outreach
}

\author{
Michael W Ross, ${ }^{1}$ Joyce Nyoni, ${ }^{2}$ Anne M Bowen, ${ }^{3}$ Mark L Williams, ${ }^{4}$ \\ John J Kashiha ${ }^{2}$
}

To cite: Ross MW, Nyoni J, Bowen AM, et al. Sexual and geographic organisation of men who have sex with men

in a large East African city: opportunities for outreach. BMJ Open 2012;2:e001813. doi:10.1136/bmjopen-2012001813

- Prepublication history for this paper are available online. To view these files please visit the journal online (http://dx.doi.org/10.1136/ bmjopen-2012-001813).

Received 19 July 2012 Accepted 25 October 2012

This final article is available for use under the terms of the Creative Commons Attribution Non-Commercial 2.0 Licence; see http://bmjopen.bmj.com

${ }^{1}$ School of Public Health, University of Texas, Houston, Texas, USA

${ }^{2}$ Department of Sociology and Anthropology, University of Dar es Salaam, Dar es Salaam, Tanzania

${ }^{3}$ School of Nursing, University of Wyoming, Laramie, Wyoming, USA ${ }^{4}$ School of Public Health, Florida Atlantic University, Coral Gables, Florida, USA

Correspondence to Dr Michael W Ross; Michael.W.Ross@uth.tmc.edu.

\section{ABSTRACT}

Objectives: To describe geographical and dispersion patterns of men who have sex with men (MSM)-related venues in a large East African city and their associations with times, participants and venue type. Methods: Mapping of MSM sites in Dar es Salaam was carried out by community research workers who catalogued, observed and reported data on venue sites, formality, times of operation, type of participant, police or vigilante activity, length of operation and the degree to which it is known both in and outside the MSM and gay communities.

Results: There is a large and widely disseminated MSM/gay satellite cultures of at least 98 sites, which has some formal sites, but is largely informal and operates within mixed entertainment environments and at particular times (including some weekend-only locales) across the city. There is a mix of places for sexual contact, largely social venues and sex on location sites. Cruising appears to be limited to open spaces and parks, with no vehicular component and almost no internet component. They are widely disseminated across all suburbs and there is no central location for MSM activities. MSM sex workers (SWs) operate at a third of these sites.

Conclusions: There is a large number of 'local' MSM contact, social and sex sites and any work with MSM will have to include these less-formal and less-known sites. The widely disseminated nature of the MSM sites, however, also suggests that sexual networks may not be closely linked between sites. The climate of stigma, abuse and potential violence appear to be limiting the development of more formal sites. This pattern is probably typical of other large urban areas in East Africa and perhaps across sub-Saharan Africa (SSA).

The concept of geographical organisation of MSM and gay communities has largely been researched in Western societies, especially in public health contexts. There is a dearth of literature on MSM and gay spatial organisation in Africa, and it is not known whether the organisation of gay and MSM

\section{ARTICLE SUMMARY}

Article focus

- Given high men who have sex with men (MSM) HIV rates in sub-Saharan Africa (SSA), it is crucial to understand how to access MSM populations for outreach and testing.

- Little is known about the spatial, temporal and contextual organisation of urban MSM in SSA.

- High stigma and discrimination against MSM impacts the form and distribution of HIV-related prevention, testing and treatment efforts in the community.

\section{Key messages}

- There is a large number of relatively small sites, nearly 100, that may serve MSM at any one time in a large East African city.

- These sites are not concentrated but are spread widely across all suburbs and usually informal, often with MSM sex workers (SWs) and female SWs operating in concert.

- MSM-related sites may be very time-specific or day-specific, range across multiple venue types and frequently be organised in such a way as to be obvious only to other MSM.

Strengths and limitations of this study

- This is one of the first sub-Saharan African studies of MSM to explore the type and distribution of MSM-associated sites.

- It may be limited to places known by informants more active in the MSM subculture and thus underestimate numbers of sites and overestimate the better known or larger sites.

- The data suggest that MSM outreach in sub-Saharan Africa (SSA) is both possible and widely geographically accessible, but that there are multiple small foci and little or no centralisation.

communities follows similar patterns to other regions. Where there are high levels of stigmatisation and discrimination (including violence), organisation may be highly informal 
or underground. Where location and organisation are key to providing targeted health information and outreach to MSM populations, with regard to HIV and STIs (sexually transmitted infections) prevention, understanding the spatial and temporal organisation of MSM and gay men in urban areas is of a particular importance. Location is also a key variable in the structure of sexual networks, which in turn determine HIV and STI transmission patterns.

Sexual networks have a number of characteristics, and HIV transmission within networks varies by their characteristics. ${ }^{1-3}$ An important structure of sexual networks in which HIV/STIs are endemic is geographic distance. ${ }^{3}$ Individuals in sexual networks with endemic HIV or sexually transmitted diseases (STD) tend to live in proximity to other network members. Proximity of sexually related sites may also facilitate sexual contact within and across networks.

A second way of conceptualising sexual context is geographic location. ${ }^{4}$ Geographic location is a fixed global place. Studies have found that HIV infections tend to cluster geographically with incidences of infection greater in some geographic locations than in others. ${ }^{5-8}$ Within sub-Saharan Africa (SSA), infections tend to be more concentrated along major ground transportation networks. ${ }^{6}$

The third conceptualisation of social context is closely related to both social networks and geographic location as a sexual venue. Sexual venues are places where individuals congregate to meet sex partners, and are associated with higher risk of HIV infections. ${ }^{7-13}$ Sexual venues bring individuals and sexual networks together, concentrating both in specific geographic locations. ${ }^{2}$ The primary purpose of a sexual venue is not always to facilitate meeting sex partners. Oftentimes the purpose of a sexual venue is socialisation. Sexual partnering may be a secondary or even unintentional outcome of visiting sexual venues. Studies have found individuals who frequent commercial sex venues, such as bars or bathhouses, public sex venues, such as parks or cruising areas or special events, such as circuit parties, are at an increased risk of HIV infection.

The concept of sexual market has also been central to the understanding of gay and MSM geographic organisation in urban areas. Ellingson and Schroeder ${ }^{14}$ identified two general types of sexual markets: transactional (finding sexual partners for short-term encounters); and relational (establishing sexual relationships through friendship and acquaintance networks). They note that manifestations of 'gay space' in large USA cities may take form of bars and clubs, bathhouses, bookstores and cruising areas. Historically, such spaces have operated on the margins of society or in urban enclaves where a so-called 'deviant' behaviour was more tolerated. Given the high stigmatisation of homosexual behaviour, such spaces also needed to operate in ways that avoided detection and control by police or vigilante groups. External stakeholders (such as family, work, religion, social and service providers) may also shape the spatial organisation of gay and MSM interactions.

High social stigmatisation of homosexuality and discrimination may suppress the development of an institutionalised gay subculture. Such social development will have spatial implications for the popularity and the visibility and location of meeting places, both transactional and relational. Stigmatisation and discrimination will also have profound implications for the development of any gay and/or MSM community or satellite culture (a term preferred to describe the organisation without assuming that they constitute a community) in a dynamic interaction between visibility, space, gay culture and legal and structural discrimination.

Ross $e t a l,{ }^{15}$ noted stages in the evolution of aggregations of quasi-isolated individuals into members of a selfconscious and interacting community. They described the stages of development from an informal stage of the organisation of places for sexual contact, to the formal stage where bars, bathhouses and other specific sites for MSM sexual contact may grow, as development of relational as well as of transactional marketplaces. Their third stage is the development of formal organisations for sexual contact and a developing sense of community follows. The fourth stage is development of civil rights services, the fifth stage the acquisition of gay-specific media and services, and the sixth stage, development of professional and recreational organisations for gay people.

This staging has implications for sexual and geographic organisation. In the first stage, it would be informal and related to cruising sites and other places for (largely anonymous) sexual contact. In the second stage, social meeting places would develop (such as non-gay bars and clubs but with some gay clientele), and then bars that are identified as predominantly or exclusively gay. Then, social and human rights support organisations tend to emerge. Thus, places identified as 'gay' are likely to develop from sex on site and cruising areas to social contact sites and then to more formal gay or predominantly gay locales (usually starting with bars and clubs that may have larger gay clientele on weekends or specific nights). This is also dependent on the level of legal and political stigmatisation, police and vigilante activity and the human rights climate. Usually, specifically gay/MSM formal locales develop in entertainment and red light areas first, as Ellingson and Schroeder ${ }^{14}$ note.

We carried out a mapping study in Dar es Salaam, Tanzania, a city of approximately 3 million population, to identify places that were associated with MSM to both identify locations in which sexual networks build up in MSM, and to identify locations where HIV-related and STI-related prevention outreach activities may be conducted. Dar es Salaam appears from our outreach work and community observations to be in the second stage of the development of gay subculture model, and is beginning to move into the third stage. While there are 
a large number of MSM/gay men in Dar es Salaam who can be fairly easily accessed, ${ }^{16}$ little is known about the spatial and functional organisation of the gay/MSM satellite culture there for HIV outreach and prevention.

\section{METHODS AND ANALYSIS}

We surveyed all identifiable gay-related and/or MSM-related sites in the Dar es Salaam extended metropolitan area. A research assistant with long-term links to the MSM subculture identified, through key informant (KI) interviews, observation and reference to the internet and gay guides, all identifiable sites and visited each site. KIs were the source of more than $80 \%$ of the information. KIs were identified through popular opinion leaders, who came from within the sites/MSM community and who know their community very well, and also through allies, MSM networks and individuals. At each site, the research assistant noted the GPS coordinates, the nature of the site and the activity, and any comments by KIs or MSM/gay men at the site. While responses varied widely, during analysis, information from KI interviews followed the data collection form themes. Information sources included Incident Reports from the community/KI, Case Reports from KI/stakeholders, survey data, observations by KI, literature reviews from other researchers and evidence (experience, observed/face-to-face discussion/calling a meeting with community/stakeholder).

The mapping and observation was carried out through KI interviews (including popular opinion leaders) for those sites which were contacted directly by telephone to make an appointment for the visit and to get brief information about the place, time and the rules and regulations of the place before arriving there, discussing with different people at that site, visiting the site at the active time(s) and information from the KI of the site. During the interviews notes were taken and these notes served as the basis for creating the interview summaries. The duration of the interviews was between 30 and $60 \mathrm{~min}$.

Data collected on the form included: Type of place (eg, bar, park, corner and stroll); Hours active? (eg, daytime, evening and night); Days active? (eg, weekends); Any sex workers (SWs) present?; Sex on site? (eg, alleys, bushes and back room); If sex on site, type of sex?; Police presence?; Vigilante presence?; Local participants or city-wide?; type of participants? (eg, younger, professional, older, married, tourists, students, military, etc); Map reference (for mapping: need not be exact for confidentiality purposes). These themes were covered in the $\mathrm{KI}$ interviews.

These data were entered into SPSS V.19 for analysis. The analysis consisted of mapping of sites by nature of activity; cross tabulations $\left(\chi^{2}\right.$ with Yates correction for discontinuity, by nature of site, formality, hours of activity, presence of SWs and law enforcement and vigilante activity) and one-way ANOVAs (means and SDs for number of participants by hour and time the site had been known as an MSM contact site).
The study was approved by the IRBs of the University of Texas and the Tanzanian National Medical Research Institute. The study's Community Advisory Board was composed of local gay men in Dar es Salaam identified by public opinion leaders and several of the researchers from prior studies in the community, with the more articulate and activist ones from a range of groups selected. They approved the data for publication. Because of the risk that any map would provide a focus for press, vigilante and police activity, we present the suburbs randomised in table 1 with a simple breakdown of MSM activities.

\section{RESULTS}

Ninety-eight sites where MSM meet and congregate in Dar es Salaam were identified. Sites ranged from walks within parks to bars and clubs. Mapping of MSM-identified sites (table 1) shows sites are spread throughout metropolitan Dar es Salaam area suburbs. The largest number of sites is in the inner suburbs rather than the central city. Spread between contact sites, social sites and sex on site locations appear relatively even, and the distribution does not differ significantly by area. There is no 'focus' of MSM/gay sites, but a range of sites appears in most areas of the city.

Of the 98 sites logged and observed, 80 were commercial premises (any place where people were carrying out business activities, including bars, shops and other commercial locations); eight parks, benches or open areas; nine non-commercial premises and one 'other' (a ghetto, or drug injection area). Eleven were formally known as gay sites; 33 known to the public as gay sites; 32 known to MSM citywide as MSM/gay sites; and 22 known only locally. Hours of activity were predominantly (table 2) evening (21\%) and night-time (36\%), but

\begin{tabular}{cccc}
\multicolumn{5}{c}{ Table 1 } & Distribution of MSM sites in Dar es Salaam \\
\hline \multicolumn{5}{c}{ Contact place } & Social site & Sex on site \\
\hline Inner residential and central suburbs \\
Suburb 1 & 4 & 2 & 0 \\
Suburb 2 & 9 & 2 & 6 \\
Suburb 3 & 4 & 0 & 1 \\
Suburb 4 & 3 & 5 & 7 \\
Suburb 5 & 1 & 4 & 2 \\
Suburb 6 & 2 & 1 & 0 \\
Suburb 7 & 6 & 2 & 0 \\
Suburb 8 & 5 & 6 & 2 \\
Outer residential suburbs & & \\
Suburb 9 & 1 & 1 & 1 \\
Suburb 10 & 1 & 0 & 2 \\
Suburb 11 & 4 & 3 & 0 \\
Suburb 12 & 1 & 1 & 0 \\
Suburb 13 & 2 & 2 & 0 \\
Suburb 14 & 0 & 2 & 0 \\
Suburb 15 & 0 & 3 & 0 \\
Total & 43 & 34 & 21 \\
\hline
\end{tabular}




\begin{tabular}{|c|c|c|}
\hline \multirow[t]{4}{*}{ Type of site } & Commercial premises & 80 \\
\hline & Park/bench/open area & 8 \\
\hline & Non-commercial premises & 9 \\
\hline & Other (ghetto) & 1 \\
\hline \multirow[t]{4}{*}{ Formality } & $\begin{array}{l}\text { Formal acknowledgement } \\
\text { of gay clientele }\end{array}$ & 11 \\
\hline & Known to public & 33 \\
\hline & Known to MSM citywide & 32 \\
\hline & Known only locally & 22 \\
\hline \multirow[t]{5}{*}{ Hours of activity } & Daytime & 7 \\
\hline & Evening & 21 \\
\hline & Night-time & 35 \\
\hline & $24 \mathrm{~h}$ & 23 \\
\hline & Specific hours & 11 \\
\hline \multirow[t]{3}{*}{ Nature of site } & Contact place & 46 \\
\hline & Social place & 32 \\
\hline & Sex on site & 20 \\
\hline \multirow{4}{*}{$\begin{array}{l}\text { Approximate players per } \\
\text { hour }\end{array}$} & $3-6$ & 8 \\
\hline & $7-12$ & 48 \\
\hline & $13-18$ & 30 \\
\hline & $19-30$ & 10 \\
\hline \multirow{2}{*}{\multicolumn{2}{|c|}{$\begin{array}{l}\text { Sex workers (SWs) } \\
\text { present } \\
\text { Vehicle cruising }\end{array}$}} & 30 \\
\hline & & 0 \\
\hline \multicolumn{2}{|l|}{ Social meeting } & 17 \\
\hline \multicolumn{2}{|l|}{ Entertainment at club-bar } & 56 \\
\hline \multicolumn{2}{|l|}{ Tourists } & 5 \\
\hline \multicolumn{2}{|l|}{ Hotel/guest house } & 7 \\
\hline \multicolumn{3}{|l|}{ Restaurant/saloon } \\
\hline \multirow[t]{4}{*}{ Law enforcement activity } & Regular & 3 \\
\hline & Occasional & 2 \\
\hline & Sporadic & 1 \\
\hline & None & 91 \\
\hline \multirow[t]{4}{*}{ Vigilante activity } & Regular & 4 \\
\hline & Sporadic & 3 \\
\hline & None & 72 \\
\hline & Unknown & 11 \\
\hline \multirow[t]{3}{*}{ Source of information } & Key informant & 82 \\
\hline & Rumour & 14 \\
\hline & Gay guide/internet & 2 \\
\hline
\end{tabular}

How long known for gay/MSM activity: $n=75$, range $2-33$ years, mean 11.4, median $11, \mathrm{SD}=6.3$.

${ }^{*} n=98$ : $n$ can be read as approximate percentage.

nearly a quarter was active $24 \mathrm{~h}$. Some ${ }^{10}$ had specific 'gay' nights, usually on weekends (Friday through Sunday). The majority were places where sexual contact could be made, with a fifth being recorded as sex on site locales, and a third as places for primarily social contact. More than half of the sites $(57 \%)$ were identified as places where entertainment was provided. The approximate number of persons at an active site ranged from 3 to $30 / \mathrm{h} \quad($ mean $=12.0$, median $=11, \mathrm{SD}=4)$. In nearly a third of the places, SWs were reported to be present. No areas for vehicle cruising were reported. No internet sites were identified. 'Other' categories identified as MSM-associated sites included hotels and guest houses where MSM could have sex, a restaurant and a saloon.
Law enforcement activity was reported as regular or occasional at five sites, and sporadic at another one. Vigilante activity was reported as regular at three sites and sporadic at another two.

Data break down by number of persons present in site per hour revealed significant differences across sites $(\mathrm{F}=9.7, \mathrm{df}=3, \mathrm{p}=0.001)$. Formal sites had the least number of persons present per hour $($ mean $=8)$, than places known to the public as gay (mean=13.6), known only to MSM citywide (mean=13.8) and places known only to local MSM (mean=9.0). There was no significant difference by hours of activity, nature of site or presence or absence of SWs, or presence of law enforcement or vigilante activity. Cross tabulation by hours of activity indicated that those with night-time or 24-hour activity were more likely to be open or park areas $\left(\chi^{2}=39.6\right.$, $\mathrm{df}=12, \mathrm{p}=0.001)$. There was a trend $(\mathrm{p}=0.08)$ for SWs to be more likely to frequent open or park areas. Law enforcement activity was also significantly more likely to occur in open or park areas $\left(\chi^{2}=33.0, \mathrm{df}=12, \mathrm{p}=0.001\right)$, but not vigilante activity.

Observations recorded during site visits about a site presented a number of informative observations, including sexual activity: 'About one week ago two people had been seen doing sex on this site'. Many referred to sex workers: 'During weekday MSM sex workers are available there'; 'Place for sex workers'; She used to sell MSM who are SW'; 'The owner of the place (name) used to sell MSM who are sex workers'; and 'This place is safe for MSM, and through this place MSM sex workers'.

Danger at several sites was also noted, 'During our visit to this site we saw policemen chasing MSM and female sex workers'; and 'Some of MSM reported that in 2009 one of MSM was beaten by community till he died there'. Many observations related to specificity of sites and the nature of the people in the site: 'Mixed of different people'; 'Most tourists like this place'; 'MSM who are Indian meet here'; 'MSM who are police meet here'; 'Most MSM who come here are hiding their sexual orientation because of the stigma from the community'; and 'Place where aged MSM come and meet other friends'.

Other observations were about the nature of the commercial site: 'Good Place for MSM, but when it comes for MSM case they increase the price of dancing room'; 'MSM meet when the band is performing'; and 'Owner of bar is a very supportive member of LGBT (lesbian, gay, bisexual and transgender) cause. He is also gay'. Sites which were sources of information were also noted: 'Place for MSM to communicate with each other; 'Ideas, share life history, health information'; and 'Source of information for Kibao Kata (dance) ceremonies, funerals, MSM issues'.

\section{DISCUSSION}

These data represent one of the few analyses of geographical sites where MSM meet, and their spatial 
distribution, in a large sub-Saharan African urban area: nearly 100 sites were identified. Geographic distribution of these sites suggests that MSM/gay activity in Dar es Salaam is not focused in particular districts or in a redlight or central city area, but relatively evenly distributed throughout the city. It is thus locally focused and suggest that from this pattern to cater to local needs. The distribution by type of site (contact, social, sex on site) suggest that MSM activity is relatively random by area with no focus, and that Dar es Salaam is probably at the second stage of development of gay community in Ross et $a l$ s staging model. ${ }^{15}$ There are formally gay sites, but the majority are mixed or specific to particular times, such as weekends. There is a relatively even spread, in addition to the small proportion $(11 \%)$ of formal sites, of those known to the public, to MSM and just known locally. MSM and gay activity is thus fairly decentralised in place and in time, and includes sites in outer suburban areas as well as inner and central areas. Those sites known (but not widely known) to some members of the public as having a gay clientele are in many cases owned by gays and sometimes owners have links with the sex industry. KIs reported that local accommodations, possibly including financial ones, with security and other authorities may be made: we have no way of judging the validity or otherwise of this information.

Approaches to social space as 'sexualised' are frequently described as heterosexualised in western contexts. ${ }^{17}$ Oswin, however, ${ }^{18}$ criticised this as an artificial divide, especially where identification as heterosexual or homosexual is frequently not associated with homosexual acts in MSM. This is particularly relevant in most sub-Saharan African contexts where any suggestion that someone is homosexual may lead to violence, blackmail or death and all spaces are implicitly regarded as heterosexual. For this reason, almost all places in Dar es Salaam are mixed and appear heterosexual to all but the most aware. Further, our data suggest that space is heavily modified by time (day vs night, week vs weekend) in its use.

Data also cast some light on the organisation, rudimentary though it is, in MSM/gay men in Dar es Salaam. Most are commercial premises, mixed (although a significant number may be 'gay' only on weekends or specific nights, which will help cover the clientele) and over half are entertainment premises including music halls (with bands or singers). Our previous work ${ }^{19}$ noted that most contacts are made in music halls, bars and in the local home environment. In such sites, men may attend without being labelled and subtle MSM mixing and careful contacts may be made. Some sites are already noted for being active in information dissemination, and might be utilised for provision of health-related material or contacts. Others are noted for having owners who are supportive of LGBT people and who may also be useful points of outreach and support. Further, there is evidence that there is some sub-specialisation (in terms of sites catering to specific groups) developing.
Open ('cruising') sites tend to be 24-h or night-time sites and also frequented by SWs, although it is apparent that there is a large and well-distributed SW industry and that SWs may be present in about a third of sites. In some sites, the management is actively involved in the provision of SW services. These data are also consistent with figures from Mombasa, about 200 miles north in Kenya, where Geibel $e t a l^{20}$ reported a large (over 700) population of MSM at 77 sites selling sex to local MSM in that city with a population of a little less than a million. Extrapolating their figures to Dar es Salaam, we might expect up to $2400 \mathrm{MSM}$ SWs in the metropolitan area. Of interest is also what has not occurred in the city. There is no evidence of vehicle cruising, probably due to the low per-capita ownership of vehicles and frequent traffic jams, and the need for careful discussion before a 'pick-up' to reduce risk for violence or blackmail. In a previous study of MSM in Dar es Salaam, ${ }^{19}$ we found that there was almost no $(<1 \%$ of contacts) internet meeting.

These data suggest that while there are a large number of identifiable gay/MSM sites, these are largely informal, and that the MSM community is still in the early stages of development, probably largely due to the strong stigma and the still very homophobic legal climate (up to life imprisonment for homosexual acts between men). ${ }^{21} \mathrm{We}$ concur with Moen $e t a l^{16}$ that there is a large and diverse group of MSM in Dar es Salaam who are relatively easy to reach by working with the MSM/gay community. Clearly, there is a variety of sites and site-based approaches offer an excellent opportunity for site and time-based recruitment and outreach. This last point is crucial to implementing effective HIV-prevention and STD-prevention programmes. However, given the lack of focus of locations, it is also essential to work locally rather than assuming that there are a limited number of locales that will access the population. It is also apparent that MSM in Dar es Salaam operate in a climate of hostility from the public, with vigilante activity and murders reported, and police activity also reported (although disproportionately focused on the more open sites). For many who are members of the gay/MSM satellite culture, there are significant risks and that outreach work in this community will have to take into account the context of stigma, fear and violence within which they operate.

Mapping of MSM-identified sites has implications for the development of interventions which focus on places rather than persons. Focusing on places recognises HIV infections $^{5-8}$ and risk behaviours ${ }^{71} 13$ tend to cluster in geographic areas, which are oftentimes associated with specific locales where people meet for sex or to socialise. ${ }^{22}$ Rather than attempting to intervene with MSM individually, focusing risk-reduction efforts can target places for interventions. Place interventions are able to target sexual networks of MSM, which has the advantage of potentially reaching large numbers of men and men who may not identify as gay or bisexual. Interventions focused on places need to be sensitive to concerns 
about stigmatisation and discrimination. However, developing culturally sensitive methods for intervening should be researched and developed.

These data are subject to several limitations. First, data are derived from those who use and know MSM-related sites and are thus biased towards those 'known' in the subculture, and will underestimate the numbers of sites and overestimate those better known. Those sites only known to a few individuals will probably not be represented. Sites that are rarely accessed or the most clandestine will be underrepresented. Second, sites are subject to change over time and possibly change related to ownership and official activity.

We present evidence for large and widely disseminated MSM/gay satellite cultures in Dar es Salaam, which have some formal sites but are largely informal and operate within mixed entertainment environments and at particular times across the city. There is a mix of places for sexual contact, largely social venues and sex on location sites. Cruising appears to be limited to open spaces and parks, with no or minimal vehicular component. There is a significant number of 'local' MSM contact, social and sex sites as well and any work with MSM will have to include these less-formal and less-known sites. The widely disseminated nature of the MSM sites, however, also suggests that sexual networks may not be closely linked between sites. While there is evidence of a greater organisation within the satellite culture, it occurrs in a climate of stigma, abuse and potential violence, which appear to be limiting it. We believe this pattern is probably typical of other large urban areas in East Africa and perhaps across SSA.

Contributors MWR wrote the manuscript, assisted by JN and MLW. AMB contributed to the manuscript and carried out the geographic analyses. JN and JJK supervised the field research. All authors edited and revised the manuscript.

Funding This study was funded by a grant from the US National Institute of Mental Health, 5R21MH090908, to the first author.

Competing interests None.

Ethics approval Tanzanian Institute for Medical Research and University of Texas Approval Number HSC-SPH-10-0033.

Provenance and peer review Not commissioned; externally peer reviewed.

Data sharing statement Data will be placed in the public domain at the conclusion of the study. We will not share any GPS information for confidentiality and protection reasons.

\section{REFERENCES}

1. Potterat J, Muth S, Rothenberg R, et al. Sexual network structure as an indicator of epidemic phase. Sex Transm Infect 2002;78: i52-8.

2. De $\mathrm{P}$, Singh $\mathrm{A}$, Wong $\mathrm{T}$, et al. Sexual network analysis of a gonorrhea outbreak. Sex Transm Infect 2004;80:280-5.

3. Rothenberg R, Muth S, Malone S, et al. Social and geographic distance in HIV risk. Sex Transm Dis 2005;32:506-12.

4. Gould P. Geographic dimensions of the AIDS epidemic. Profess Geogr 1989;41:71-8.

5. Latkin C, Glass G, Duncan T. Using geographic information systems to assess spatial patterns of drug use, selection bias and attrition among a sample of injection drug users. Drug Alcohol Depend 1998:50:167-75.

6. Ferguson A, Morris C. Mapping transactional sex on the northern corridor highway in Kenya. Health Place 2007;13:504-19.

7. Khan M, Rasolofomanana J, McClamroch K, et al. High-risk sexual behavior at social venues in Madagascar. Sex Transm Dis 2008;35:738-45.

8. Uchudi J, Magadi M, Mostazir M. A multilevel analysis of the determinants of high-risk sexual behavior in sub-Saharan Africa. J Biosociol Sci 2012;44:289-311. doi: 10.1017/ S0021932011000654.

9. Colfax G, Mansergh G, Guzman R, et al. Drug use and sexual risk behavior among gay and bisexual men who attend circuit parties: a venue-based comparison. J Acquir Immune Defic Syndr 2001;28:272-379.

10. Parsons J, Halkitis P. Sexual and drug-using practices of HIV-positive men who frequent public and commercial sex environments. AIDS Care 2002;14:815-26.

11. Horvath K, Bowen A, Williams M. Virtual and physical venues as contexts for HIV risk among rural men who have sex with men. Health Psychol 2005;25:237-42.

12. Halkitis P, Palamar J, Mukherjee P. Poly-club-drug use among gay and bisexual men: a longitudinal analysis. Drug Alcohol Depend 2007;89:153-60.

13. Grov C, Golub S, Parsons J. HIV status differences in venues where highly sexually active gay and bisexual men meet sex partners: Results from a pilot study. AIDS Educ Prev 2010;22:496-508.

14. Ellingson S, Schroeder K. Race and the construction of same-sex markets in four Chicago neighborhoods. In: Laumann EO, Ellingson S, Mahay J, Paik A, Youm Y.eds. The sexual organization of the city. Chicago: University of Chicago Press, 2004:93-123.

15. Ross MW, Fernández-Esquer ME, Seibt A. Understanding across the sexual orientation gap. In: Landis D, Bhagat RS.eds. Handbook of intercultural training. 2 edn. Thousand Oaks, CA: Sage, 1996;414-30.

16. Moen $\mathrm{K}$, Aggleton $\mathrm{P}$, Leshabari $\mathrm{M}$, et al. Not at all so hard to reach: same-sex attracted men in Dar es Salaam. Cult, Health Sexuality 2012;14:195-208. doi: 10.1080/13691058.2011.632825

17. Kirby S, Hay I. (Hetero) sexing space: Gay men and 'straight' space in Adelaide, South Australia. Prof Geogr 1997;49:295-305.

18. Oswin N. Critical geographies and the uses of sexuality: deconstructing queer space. Progr Hum Geogr 2008;32:89-103.

19. Nyoni J, Ross MW. Condom use and HIV testing in urban Tanzanian men who have sex with men. AIDS Care 2012. doi:10.1080/ 09540121.2012 .699671

20. Geibel S, van der Elst EM, King'ola N, et al. 'Are you on the market?': a capture-recapture enumeration of men who sell sex to men in and around Mombasa, Kenya. AIDS 2007;21:1349-54.

21. Wikipedia. 2012. http://en.wikipedia.org/wiki/LGBT_rights_in_ Tanzania. (accessed 25 Jan 2012)

22. Wyle J, Jolly A. Patterns of chlamydia and gonorrhea infection in sexual networks in Manitoba, Canada. Sex Transm Dis 2001;28:14-24. 